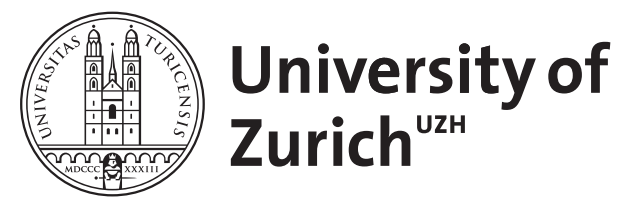

\title{
Management of traumatic brain injury
}

\author{
Imhof, Hans-Georg ; Lenzlinger, Philipp M
}

\begin{abstract}
In the past years several recommendations have been published concerning the diagnostic work-up and treatment of patients with traumatic brain injury (TBI). They show that with regard to the surgical management of acute epidural hematomas, acute subdural hematomas, traumatic parenchymal lesions, posterior fossa mass lesions, as well as depressed skull fractures there is a lack of controlled studies, which would allow to define standards of treatment or guidelines, respectively. Nonetheless, treatment protocols serve an important purpose, because they may improve management of TBI patients by promoting uniform decision-making in the treatment of these patients, namely in • the identification of the few patients likely to suffer from complications among the large number of patients who sustain a mild to moderate head injury and - strategies for avoiding posttraumatic cerebral ischemia. In this context, the authors focus on the importance of plain skull X-rays and CT scan, respectively, in the workup of mild TBI patients and on the indications for decompressive craniectomy for the relief of intractable elevation of intracranial pressure following severe TBI
\end{abstract}

DOI: https://doi.org/10.1007/s00068-005-2061-5

Posted at the Zurich Open Repository and Archive, University of Zurich ZORA URL: https://doi.org/10.5167/uzh-156586

Journal Article

Published Version

Originally published at:

Imhof, Hans-Georg; Lenzlinger, Philipp M (2005). Management of traumatic brain injury. European Journal of Trauma, 31(4):331-343.

DOI: https://doi.org/10.1007/s00068-005-2061-5 


\title{
Management of Traumatic Brain Injury
}

\author{
Application of Guidelines for Diagnostics and Therapy
}

\author{
Hans-Georg Imhof', Philipp M. Lenzlinger ${ }^{2}$
}

\begin{abstract}
In the past years several recommendations have been published concerning the diagnostic work-up and treatment of patients with traumatic brain injury (TBI). They show that with regard to the surgical management of acute epidural hematomas, acute subdural hematomas, traumatic parenchymal lesions, posterior fossa mass lesions, as well as depressed skull fractures there is a lack of controlled studies, which would allow to define standards of treatment or guidelines, respectively. Nonetheless, treatment protocols serve an important purpose, because they may improve management of TBI patients by promoting uniform decision-making in the treatment of these patients, namely in

- the identification of the few patients likely to suffer from complications among the large number of patients who sustain a mild to moderate head injury and - strategies for avoiding posttraumatic cerebral ischemia.

In this context, the authors focus on the importance of plain skull X-rays and CT scan, respectively, in the work-up of mild TBI patients and on the indications for decompressive craniectomy for the relief of intractable elevation of intracranial pressure following severe TBI.
\end{abstract}

\section{Key Words}

Traumatic brain injury · Management - Guidelines . Decompressive craniectomy - Computed tomography · Plain skull X-ray

Eur J Trauma 2005;31:331-43

DOI 10.1007/s00068-005-2061-5

\section{Introduction}

Guidelines are published in an effort to promote best practice, and to optimize the care of patients. They have to be based on the best available scientific, evidence-based methodology and must incorporate the latest developments and evidence on the care. They are also designed to minimize variations in practice, by bringing everyone up to best performance. It has been shown that adherence to a protocol based on guidelines may significantly improve mortality and outcome $[14,32,119]$.

The development of guidelines may follow different approaches: either evidence-based as chosen by the Brain Trauma Foundation [15] or, where there is a lack of high-quality evidence, consensus- and expert opinion-based as developed by the European Brain Injury Consortium (EBIC) [72].

Evidence-based guideline development links recommendations directly to scientific evidence of effectiveness; rules of evidence are emphasized over expert opinion in making recommendations $[15,125]$. The classification of evidence into three categories leads to the formulation of recommendations called Standards, Guidelines, and Options (Table 1). This terminology was developed to indicate the strength of the recommendations based upon the strength of scientific medical evidence.

In the past years, several guidelines have been published regarding the treatment and the diagnostic work-up of patients suffering from traumatic brain injury (TBI) [1, $5,6,8,15,51,72,103,115,121]$. They show that with regard to the surgical management of acute epidural hematomas, acute subdural hematomas, traumatic parenchymal lesions, posterior fossa mass lesions, as well as

'Department of Neurosurgery, University Hospital of Zurich, Switzerland,

${ }^{2}$ Division of Trauma Surgery, University Hospital of Zurich,

Switzerland.

Received: May 31, 2005; accepted: June 22, 2005. 
Table 1. Brain Trauma Foundation: surgical management of traumatic brain injury (Bullock et al. [15]).

Relation of strength of evidence to strength of recommendations

Class I Evidence is used to support treatment recommendations of the strongest type, practice Standards, reflecting a high degree of clinical certainty. Requires at least one randomized controlled trial as part of a body of literature of overall good quality and consistency addressing the specific recommendation (evidence levels Ia, Ib)

Class II Evidence is used to support Guidelines, reflecting a moderate degree of clinical certainty. Requires the availability of well-conducted studies but not randomized evidence on the topic of the recommendation (evidence levels II, IIa, IIb, III)

Class III Evidence supports practice 0 ptions, reflecting unclear clinical certainty. Requires evidence obtained from expert committee reports or opinions and/or clinical experiences of respected authorities. Indicates an absence of directly applicable clinical studies of good quality (evidence level IV)

depressed skull fractures there is a lack of controlled studies, which would allow to define standards of treatment or guidelines, respectively. Most recommendations are based on expert opinion and clinical experience, so-called Class III recommendations or practice Options (see below). Recommendations may not necessarily be weak where the evidence is weak, especially when the logic of the recommendation and all the evidence supporting it, however weak, as well as clinical experience all support the recommendation [15]. One such example is the recommendation regarding timing of evacuation of epidural hematomas. According to the paradigm embraced and used in the "Guidelines for the management of severe traumatic brain injury" (STBI), case series indicate that patients who have a Glasgow Coma Scale (GCS) score of $\leq 8$ with evidence of a "blown pupil" and who are operated upon early on, achieve better outcomes. However, this level of evidence can only support a practice Option. Yet, no competent neurosurgeon will allow a patient in this clinical scenario to be neglected when the need for surgical relief of brain compression is so clear. It is fairly certain to say that there will never be a randomized controlled trial for this circumstance, and thus never a practice "Standard". On the other hand, there is no evidence that waiting to operate upon such a patient is beneficial, and therefore an "Option" to delay surgical evacuation will also probably never be promulgated. If, indeed, such a recommendation were put forward, it would never be accepted by the profession, and rightfully so misinterpreted.

Nonetheless, treatment protocols serve an important purpose, because they may improve management of TBI patients by promoting uniform decision-making in the treatment of these patients, thus also facilitating comparison of patient populations across treatment centers. For example, this may be of importance where we try to identify the few patients likely to suffer from complications among the large number of patients who sustain a mild to moderate head injury (GCS 13-15, and 9-12, respectively). The avoidance of secondary cerebral ischemia is another area, where guidelines seek to promote a more uniform course of action.

In this present review we will address the problem of employing the adequate diagnostic tool (skull X-ray [SR] vs. computed tomography [CT]) following mild head injury, as well as discuss the indications and timing of decompressive craniectomy (DCE) in the neurosurgical treatment of patients with TBI.

\section{Mild Traumatic Brain Injury}

Mild traumatic brain injury (MTBI) is defined as the consequence of blunt (nonpenetrating) impact with sudden acceleration, deceleration or rotation of the head with a GCS score of 13-15 [110] on admission to hospital [121]. The primary goal of initial management in MTBI is to identify the patients at risk for intracranial abnormalities and especially those who may need neurosurgical intervention.

Therefore the Neurotraumatology Committee of the World Federation of Neurosurgical Societies (WFNS) [100] has classified mild head injury in adults as

- low-risk mild head injury: those patients with a GCS of 15 and without a history of loss of consciousness, amnesia, vomiting, or diffuse headache;

- medium-risk mild head injury: those with a GCS of 15 and one or more of the following symptoms: loss of consciousness, amnesia, vomiting, or diffuse headache;

- high-risk mild head injury: those with an admission GCS of 14 or 15, with a skull fracture and/or neurologic deficits.

Patients with one of the following risk factors - coagulopathy, drug or alcohol consumption, previous neurosurgical procedures, pre-trauma epilepsy, or age $>60$ years - are included in the high-risk group independently of the clinical presentation.

An outcome study of patients who had a head injury suggested that patients with a low risk of dying - that is, patients with mild head injury - are at the greatest risk of inadequate diagnosis and treatment [58]. Most of the preventable mortality arose from late diagnosis of deterioration in patients who initially seemed to have minor inju- 
ries. About $75 \%$ of deceased patients presented with delayed intracranial hematoma [70, 71, 112]. The frequency of a surgical lesions (i.e., requiring surgical evacuation) in hospital-admitted patients varies between $0.7 \%$ and $4.0 \%$ in different studies $[104,109]$. Even if the GCS score is 15 , intracranial lesions cannot be completely excluded clinically on head trauma patients who have loss of consciousness or amnesia, regardless of age, mechanism of injury, or clinical findings [53]. This poses the question about the adequate diagnostic work-up and/or the need for in-hospital clinical surveillance of these patients. Use of a clinical decision scheme based on risk factors may facilitate this process [121].

The question whether to perform a CT scan or not is only applicable to patients who present in the emergency department with a normal neurologic exam. All patients, who do not have a GCS of 15 , or who with a GCS of 15 present with neurologic or neuropsychological deficits [120] require a CT scan. As did the Neurotraumatology Committee of the WFNS [100], the task force of the European Federation of Neurosurgical Societies (EFNS) [121] has listed the risk factors for intracranial complications following mild head injury (Table 2).

In conscious (GCS 15) and neurologically normal patients the finding of a linear fracture of the cranial vault increases the risk of intracranial hematoma considerably [76, 109]. Therefore the Society of British Neurological Surgeons [103] recommends conventional SR in two planes according to the criteria in Table 3 in order to identify patients at risk of developing a surgically significant intracranial hematoma. Indication to perform a CT scan or to transfer the patient to another hospital, therefore, was based on the presence of a skull fracture. However, more recent studies have questioned the value of conventional SR in MTBI. Hofman et al. [48] found that the probability of intracranial hematoma in patients with MTBI and skull fracture is not elevated 41 -fold [76] but only by a factor of 5 . Severe intracranial pathology may be present even in the absence of a fractured skull. Furthermore, skull factures are often missed by the less experienced physicians [111], who usually see most patients with MTBI in most institutions. This will decrease the sensitivity of the SR even further. Even in detecting skull fractures CT was found to be superior to plain X-rays [112].

The "EFNS guideline on mild traumatic brain injury" [121] therefore postulates: "Skull radiography is of insufficient value in the detection of intracranial abnormalities
Table 2. The task force of the European Federation of Neurosurgical Societies [121].

Risk factors for intracranial complications after mild traumatic brain injury

- Unclear or ambiguous accident history

- Continued posttraumatic amnesia

- Retrograde amnesia > 30 min

- Trauma above the clavicles including clinical signs of skull fracture (skull base or depressed skull fracture)

- Severe headache

- Vomiting

- Focal neurologic deficit

- Seizure

- Age $<2$ years

- Age $>60$ years

- Coagulation disorders

- High-energy accident ${ }^{c}$

- Intoxication with alcohol/drugs

${ }^{a}$ Continued posttraumatic amnesia may be interpreted as a GCS verbal reaction of 4 and hence may be defined as GCS $<15$

${ }^{\mathrm{b}}$ The Canadian CT head rule found age $>65$ to be a risk factor [105]

${ }^{c}$ According to Advanced Trauma Life Support principles, a high-energy vehicle accident is defined as initial speed $>64 \mathrm{~km} / \mathrm{h}$, major auto-deformity, intrusion into passenger compartment $>30 \mathrm{~cm}$, extrication time from vehicle $>20$ min, falls $>6 \mathrm{~m}$, roll over, auto-pedestrian accidents, or motor cycle crash $>32 \mathrm{~km} / \mathrm{h}$ or with separation of rider and bike (American College of Surgeons Committee on Trauma 2004, [8])

in patients with MTBI (Grade A, recommendation)", and " $\mathrm{CT}$ is the gold standard for the detection of intracranial abnormalities and is a safe method for home triage". Similarly, the WFNS [100] recommends obtaining a CT scan from patients with "medium-risk mild head injury". If CT scanning is not so readily available, adults should have an SR and, if this shows a fracture, should be moved to the "high-risk" category and undergo CT scanning as every patient in the high-risk category.

Table 3. Guidelines for the initial management of head injuries: recommendations from the Society of British Neurological Surgeons [103]. CSF: cerebrospinal fluid.

Indications for skull X-ray after recent head injury

Orientated patient

- History of loss of consciousness or amnesia

- Suspected penetrating injury

- CSF or blood loss from nose or ear

- Scalp laceration (to bone or $5 \mathrm{~cm}$ long), bruise or swelling

- Violent mechanism of injury

- Persisting headache and/or vomiting

- In a child, fall from a significant height (which depends in part on the age of the child)

- and/or onto a hard surface; tense fontanel; suspected nonaccidental injury

Patient with impaired consciousness or neurologic signs

All patients unless urgent $\mathrm{CT}$ is performed or transfer to neurosurgery is arranged

Note: skull X-ray is not necessary, if CT is to be performed 
A liberal policy of CT scanning is warranted for pediatric patients with a high-risk mechanism of injury despite maintenance of normal neurologic status in the field and at hospital screening [102].

When CT is available, plain $\mathrm{X}$-ray films of the skull contribute little or no additional information for the clinical management of the acute head trauma patient.

In most major trauma centers, therefore, plain X-ray films of the skull have been supplanted by CT scanning [21, 28, 68, 69], even if there is no agreement in the literature on whether patients with MTBI should undergo CT scan and be discharged home if inconspicuous, or whether all patients should be admitted but only a few undergo CT [105]. The EFNS task force [121] modified the decision schemes of the Dutch and Scandinavian guidelines [51, 115] for the initial management of MTBI (Figure 1) and recommend a CT scan over plain X-ray in MTBI, if warranted as do the WFNS guidelines [100].

\section{Conclusion}

CT scan is the gold standard for early detection of serious complications in MTBI. Only low-risk mild injury patients - those with a GCS score of 15 and without a history of loss of consciousness, amnesia, vomiting, or diffuse headache - do not need a CT. In these patients the risk of intracranial hematoma requiring surgical evacuation is definitively $<0.1: 100$ [31].

For all other MTBI patients a CT scan is highly recommended. There is no sufficient data to conclude in which patients a normal initial CT scan may allow for immediate discharge home or if in-hospital observation is necessary.

\section{Neurosurgical Interventions}

The most serious complication of TBI is the occurrence of an intracranial mass lesion, i.e., a hematoma within the skull vault, which occurs in $25-45 \%$ of severe head
Mild traumatic brain injury CGCS = 13-15)

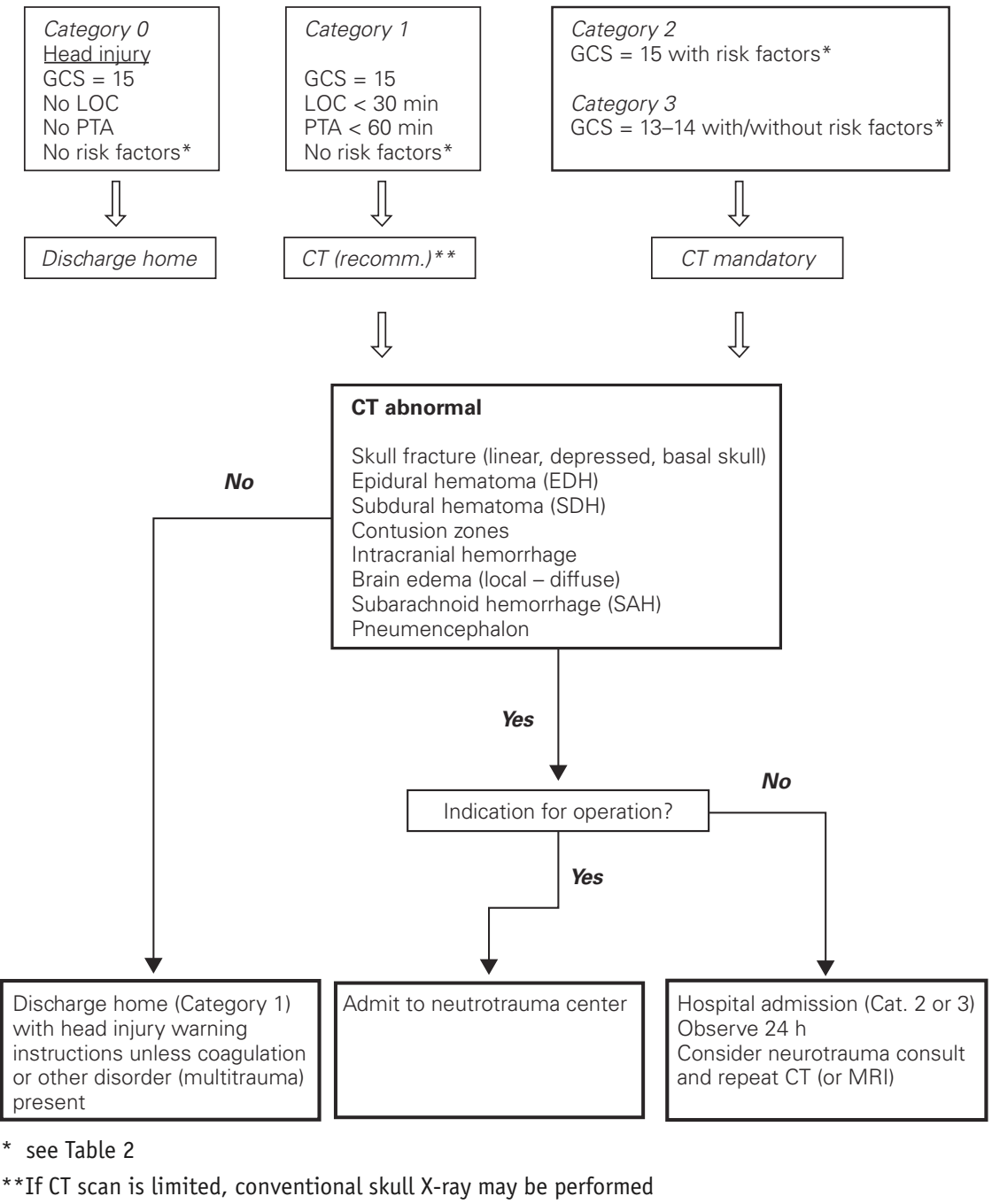

Figure 1. Management of mild traumatic brain injury (modified from the EFNS guideline on mild traumatic brain injury [121]). LOC: loss of consciousness, PTA: posttraumatic amnesia.

injuries, and in $3-23 \%$ of mild to moderate TBI cases [113]. Without effective surgical management, an intracranial hematoma may transform an otherwise benign clinical course with the expectation of recovery, into a situation where death or permanent vegetative survival is imminent.

The aim of neurosurgical care is to minimize the secondary brain damage that occurs after a severe head injury [101]. This includes the evacuation of an intracranial mass lesion, the reduction of intracranial volume and external ventricular drainage with hydrocephalus. When conservative treatment fails, a DCE might be successful in lowering intracranial pressure (ICP). 
Table 4. The Brain Trauma Foundation, the American Association of Neurological Surgeons: the Joint Section on Neurotrauma and Critical Care [10].

Indications for intracranial pressure (ICP) monitoring in adults

In adult patients ICP monitoring is appropriate with severe head injury with an abnormal admission CT scan. Severe head injury is defined as a GCS 3-8 after cardiopulmonary resuscitation. An abnormal CT scan of the head is one that reveals hematomas, contusions, edema, or compressed basal cisterns.

ICP monitoring is appropriate in patients with severe head injury with a normal CT scan, if two or more of the following features are noted at admission:

- age $>40$ years

- unilateral or bilateral motor posturing

- systolic blood pressure $<90 \mathrm{mmHg}$.

ICP monitoring is not routinely indicated in patients with mild or moderate head injury. However, a physician may choose to monitor ICP in certain conscious patients with traumatic mass lesions. ICP treatment should be initiated at an upper threshold of $20-25 \mathrm{mmHg}$. Interpretation and treatment of ICP based on any threshold should be corroborated by frequent clinical examination and cerebral perfusion pressure (CPP) data

Two patient populations must be distinguished when addressing treatment guidelines in TBI:

(A) patients who do not require surgical decompression, but who need monitoring of ICP in order to determine cerebral perfusion pressure (CPP), and

(B) patients who will undergo neurosurgical treatment.

\section{(A) Injuries, Requiring ICP Monitoring, but not Surgical Decompression}

ICP is derived from cerebral blood and cerebrospinal fluid (CSF) circulatory dynamics and can be affected in the course of many diseases of the central nervous system. Monitoring of ICP requires an invasive transducer [24].

In patient with severe head injury the correlation between high ICP and poor outcome is widely accepted $[3,71,84]$. A recent review of data from twelve Canadian trauma centers also revealed a significant association between ICP monitoring and improved survival [63].

So far, there have not been any prospective randomized trials analyzing whether ICP measurement in itself positively affects outcome following TBI. However, two class I studies $[29,82]$ and clinical experience show that ICP monitoring:

- allows the earlier detection of delayed intracerebral hemorrhage,

- allows for lowering of ICP through CSF drainage (if an intraventricular monitoring device is used),

- may avoid an uncontrolled (or unnecessary) ICP-lowering therapy with potentially severe unwanted side effects,

- may help to predict outcome.
Table 5. Guidelines for the acute medical management of severe traumatic brain injury in infants, children, and adolescents (Adelson et al. [1]).

Indications for intracranial pressure (ICP) monitoring in children

In infants and children ICP monitoring is appropriate with severe traumatic brain injury (GCS $\leq 8)$. The presence of open fontanels and/or sutures in an infant with severe traumatic brain injury does not preclude the development of intracranial hypertension or negate the utility of ICP monitoring. ICP monitoring is not routinely indicated in infants and children with mild or moderate head injury. However, a physician may choose to monitor ICP in certain conscious patients with traumatic mass lesions or in patients for whom serial neurologic examination is precluded by sedation, neuromuscular blockade, or anesthesia. Treatment for intracranial hypertension, defined as a pathologic elevation in ICP, should begin at an ICP $\geq 20 \mathrm{mmHg}$. Interpretation and treatment of intracranial hypertension based on any ICP threshold should be corroborated by frequent clinical examination, monitoring of physiologic variables (e.g., cerebral perfusion pressure), and cranial imaging

For comatose patients (GCS 3-8) with abnormal CT findings, ICP monitoring is recommended (Tables 4 and 5). Generally, comatose patients with normal CT scans have a low risk of increased ICP, however, this risk increases for patients $>40$ years or if systolic blood pressure is $<90 \mathrm{mmHg}$.

In patients with mild (GCS 13-15) or moderate TBI (GCS 9-12), no routine ICP monitoring is recommended, because these patients can be evaluated clinically. For young children the same recommendations are valid, regardless of the degree of closure of the fontanels. In patients who require ICP monitoring, a ventricular catheter (intracerebroventricular [ICV] device) connected to an external strain gauge transducer or catheter tip pressure transducer device is the most accurate and reliable method of monitoring ICP and enables therapeutic CSF drainage. Parenchymal catheter tip pressure transducer devices measure ICP similar to ventricular ICP pressure but have the potential for significant measurement differences and drift due to the inability to recalibrate. These devices are advantageous when ventricular ICP is not obtained or if there is obstruction in the fluid couple. Subarachnoid or subdural fluid-coupled devices and epidural ICP devices are currently less accurate $[10,24,36,64,127]$.

If an ICP monitor was placed in the emergency room, it can be used to assess the CPP and guide therapeutic interventions aimed at reducing ICP. If the multiply injured patient with TBI or a depressed level of consciousness is to undergo a lengthy $(>2 \mathrm{~h})$ nonneurosurgical operation without full evaluation of potential brain injury, then an ICP monitor should be placed dur- 
ing surgery to guide management, provided coagulation parameters are acceptable [67]. Clinically significant infections or hemorrhage associated with ICP devices causing patient morbidity are rare and should not deter the decision to monitor ICP [46, 49, 124].

In children and adults who are stable and who can be assessed clinically, repeated CT scans do not detect lesions requiring a change of treatment. They are therefore not required $[13,56,107)$. However, in patients with STBI and normal initial CT scan, in whom ICP is not monitored, at least one control CT scan is mandatory $[70,71]$.

ICP treatment should be initiated at an upper threshold of $20-25 \mathrm{mmHg}$ [11]. Interpretation and treatment of ICP based on any threshold should be corroborated by frequent clinical examination and $\mathrm{CPP}$ data $[30,78,85,103]$. ICP monitoring may be stopped as soon as the patient becomes clinically assessable and if within the next $24 \mathrm{~h}$ no major therapeutic intervention is scheduled. For ICV devices the amount of necessary CSF drainage should not exceed $50 \mathrm{ml} / 24 \mathrm{~h}$ before removal.

(B) Injuries Requiring Neurosurgical Decompression Prioritization of evaluation and treatment of head injuries versus systemic injuries will depend on the hemodynamic stability of the patient. The initial management of the head-injured should be similar to the polytrauma without head injury focusing on the rapid control of hemorrhage and restoration of vital signs and tissue perfusion [6] (Advanced trauma life support [ATLS ${ }^{\circledR}$ ] guidelines). Cavity decompression may be necessary as well as temporary skeletal stabilization [37]. Stable patients should undergo a CT scan of the head performed before going to the operating room for systemic injuries to evaluate any potentially operative lesions such as an acute subdural or epidural hematoma. The clinical triad of depressed consciousness, unequal pupils, and hemiparesis is diagnostic of a mass lesion. However, all three findings may not be present, or may not be detectable in the tracheally intubated patient after administration of muscle relaxant [67].

When a CT scan has to be postponed, insertion of an ICP monitoring device - after checking for adequate hemostasis [88] - may be helpful in optimizing intraoperative care for the TBI patient. Once the life-threatening injuries are stabilized, urgent head CT should be obtained. Intracranial compression must be relieved as quickly as possible in order to minimize the extent of secondary injury. When these lesions are associated with other life-threatening systemic injuries, simultaneous operations may need to be performed to achieve the best neurologic outcome.

The prognosis of intracranial mass lesions correlates directly with the time between onset of neurologic symptoms (pupillary abnormalities and/or neurologic deterioration) and the surgical evacuation of the lesion. Time from onset of coma to surgery is more important than time between trauma and surgery $[12,27,43,60$, 96,123 ]. Haselsberger et al. [43] found that in a group of patients with acute epidural hemorrhage, craniotomy for evacuation within $2 \mathrm{~h}$ led to $67 \%$ good outcomes and $17 \%$ mortality, whereas decompression $>2 \mathrm{~h}$ after onset of coma dramatically reduced good outcomes to $13 \%$ and increased mortality to $56 \%$.

There is a lack of "Standards" or "Guidelines" with regard to surgical interventions in acute TBI. However, the absolute necessity to evacuate mass lesions precludes randomized controlled trials, as mentioned before. Tables 6 to 8 list the "Options" for surgical treatment of intracranial, extracerebral hematomas and for intraparenchymal hematomas as well as for depressed skull fractures [15].

\section{Decompressive Craniectomy}

The importance of intracranial hypertension in determining the outcome of head-injured patients is well known $[9,16,77,78]$ and has been confirmed only recently by Juul et al. [55] in a large clinical trial.

Following TBI, raised ICP refractory to standard treatment measures (sedation, ventricular CSF drainage, mild hyperventilation, mannitol) is a common problem [106]. It is estimated that $10-15 \%$ of patients admitted with STBI will ultimately manifest medically and surgically intractable elevated ICP with an associated mortality of $84-100 \%$ [65, 84]. An obvious way to convert the closed box surrounding the brain into an open box is to open the skull by craniectomy, which has been performed for decades [17, 23, 38, 57, 61, 91].

In recent years there has been a renewed interest in the use of DCE [87] for control of otherwise intractable ICP in adult patients $[35,40,42,44,89]$ as well as in children $[33,45,92,95,108]$ with head injuries and severe intracranial hypertension, but no surgical mass lesion. Horizontal midline shift and compression of the basal cisterns, as demonstrated by CT scan, are well-known predictors of poor outcome $[114,117]$. There is evidence that the operation does favorably influence ICP. In both 
Table 6. Brain Trauma Foundation: surgical management of traumatic brain injury (Bullock et al. [15]).

\section{Surgical management of acute epidural hematomas}

Indications for surgery

- An epidural hematoma $>30 \mathrm{~cm}^{3}$ should be surgically evacuated regardless of the patient's Glasgow Coma Scale score

- An epidural hematoma $<30 \mathrm{~cm}^{3}$ and with $<15 \mathrm{~mm}$ thickness and with $<5 \mathrm{~mm}$ midline shift in patients with a Glasgow Coma Scale score $>8$ without focal deficit can be managed nonoperatively with serial CT scanning and close neurologic observation in a neurosurgical center

- It is strongly recommended that patients with an acute epidural hematoma in coma (Glasgow Coma Scale score < 9 ) with anisocoria undergo surgical evacuation as soon as possible

Timing

It is strongly recommended that patients with an acute epidural hematoma in coma (Glasgow Coma Scale score $<9$ ) with anisocoria undergo surgical evacuation as soon as possible

Methods

There are insufficient data to support one surgical treatment method. However, craniotomy provides a more complete evacuation of the hematoma

\section{Surgical management of acute subdural hematomas}

Indications for surgery

- An acute subdural hematoma with a thickness $>10 \mathrm{~mm}$ or midline shift $>5 \mathrm{~mm}$ on CT should be surgically evacuated, regardless of the patient's Glasgow Coma Scale score

- All patients with acute subdural hematoma in coma (Glasgow Coma Scale score $<9$ ) should undergo intracranial pressure monitoring

- A comatose patient (Glasgow Coma Scale score $<9$ ) with a subdural hematoma $<10 \mathrm{~mm}$ thickness and midline shift $<5 \mathrm{~mm}$ should undergo surgical evacuation of the lesion, if the Glasgow Coma Scale score decreased between the time of injury and hospital admission by 2 or more points on the Glasgow Coma Scale and/or the patient presents with asymmetric or fixed and dilated pupils and/or the intracranial pressure exceeds $20 \mathrm{mmHg}$

Timing

In patients with acute subdural hematoma and indications for surgery, surgical evacuation should be done as soon as possible Methods

If surgical evacuation of an acute subdural hematoma in a comatose patient (Glasgow Coma Scale score $<9$ ) is indicated, it should be done using a craniotomy with or without bone flap removal and duraplasty

animal and human studies, surgical decompression has been found to lower ICP by increasing intracranial volume $[4,7,25,38,41,42,44,47,54,61,78,89]$. Muench et al. [81] found that the mean volume gained by surgical decompression ranged from 15.9 to $347.4 \mathrm{~cm}^{3}$ with a median volume of $73.6 \mathrm{~cm}^{3}$. In subtemporal craniectomy without opening the dura, Alexander et al. [4] measured a gain of $30 \mathrm{~cm}^{3}$ in volume. Craniectomy also improves dynamics of ICP [25, 40, 42, 89, 95]. Yoo et al. [126] monitored the ventricular pressure continuously, during bilateral decompressive procedures and during the postoperative period. The initial ventricular ICP was variable, ranging from 16 to $65.8 \mathrm{mmHg}$. Immediately after bilateral craniectomy, the mean ventricular ICP decreased to $50.2 \pm 16.6 \%$ of the initial ICP (range 5$51.5 \mathrm{mmHg}$ ). Additional opening of the dura decreased the mean ICP by an additional $34.5 \%$ and reduced the ventricular pressure to $15.7 \pm 10.7 \%$ of the initial pressure (range 0-15 $\mathrm{mmHg}$ ). Ventricular pressure measured postoperatively in the neurosurgical intensive care unit was lowered to $15.1 \pm 16.5 \%$ of the initial ICP. The ventricular ICP trend in the first $24 \mathrm{~h}$ after decompressive surgery was an important prognostic factor; if it was $>35 \mathrm{mmHg}$, the mortality rate was $100 \%$. The same experience was reported by Jourdan et al. [54] in patients suffering from cerebrovascular accidents. After flap removal, ICP decreased by $15 \%$ and, after opening of the dura, it fell a further $70 \%$. In six patients they were able to carry out continued postoperative monitoring of ICP, which stayed $<50 \%$ of initial values. This decrease in ICP is sustained for hours [89]. This two-leveled drop in ICP was also noted by Jaeger et al. [52]. In that study, simultaneously, tissue oxygenation $\left(\mathrm{P}_{\mathrm{ti}} \mathrm{O}_{2}\right)$ increased rapidly from $0.8 \mathrm{kPa}(6 \mathrm{mmHg})$ to $3.07 \mathrm{kPa}$ $(23 \mathrm{mmHg}) . \mathrm{P}_{\mathrm{ti}} \mathrm{O}_{2}$ and ICP remained at noncritical ranges postoperatively. Whitfield et al. [122] reported beneficial effects of decompression also on other ICP variables, such as ICP waveform, the magnitude of slow ICP waves, and the correlation coefficient between ICP amplitude and ICP; CPP, however, was not affected. Examining the effects of the procedure on $\mathrm{CT}$ appearance, a decrease in midline shift and improved visibility of the mesencephalic cisterns were observed as a result of decompression [81].

\section{Indications}

When discussing DCE, two entirely different situations have to be distinguished [116]: 
Table 7. Brain Trauma Foundation: surgical management of traumatic brain injury (Bullock et al. [15]).

\section{Surgical management of traumatic parenchymal lesions}

Indications for surgery

- Patients with parenchymal mass lesions and signs of progressive neurologic deterioration referable to the lesion, medically refractory intracranial hypertension, or signs of mass effect on CT scan should be treated operatively

- Patients with Glasgow Coma Score (GCS) 6-8 with frontal or temporal contusions $>20 \mathrm{~cm}^{3}$ in volume with midline shift $>5 \mathrm{~mm}$ and/or cisternal compression on CT scan, and patients with any lesion $>50 \mathrm{~cm}^{3}$ in volume should be treated operatively

- Patients with parenchymal mass lesions who do not show evidence for neurologic compromise, have controlled intracranial pressure (ICP), and no significant signs of mass effect on CT scan may be managed nonoperatively with intensive monitoring and serial imaging

Timing and methods

Craniotomy with evacuation of mass lesion is recommended for those patients with focal lesions and the surgical indications listed above.

Bifrontal decompressive craniectomy within $48 \mathrm{~h}$ of injury is a treatment option for patients with diffuse, medically refractory posttraumatic cerebral edema and resultant intracranial hypertension.

Decompressive procedures, including subtemporal decompression, temporal lobectomy, and hemispheric decompressive craniectomy, are treatment options for patients with refractory intracranial hypertension and diffuse parenchymal injury with clinical and radiographic evidence for impending transtentorial herniation

\section{Surgical management of posterior fossa mass lesions}

Indications for surgery

- Patients with mass effect on CT scan or with neurologic dysfunction or deterioration referable to the lesion should undergo operative intervention. Mass effect on CT scan is defined as distortion, dislocation, or obliteration of the fourth ventricle, compression or loss of visualization of the basal cisterns, or the presence of obstructive hydrocephalus

- Patients with lesions and no significant mass effect on CT scan and without signs of neurologic dysfunction may be managed by close observation and serial imaging

\section{Timing}

In patients with indications for surgical intervention, evacuation should be performed as soon as possible, since these patients can deteriorate rapidly, thus worsening their prognosis

Methods

Suboccipital craniectomy is the predominant method reported for evacuation of posterior fossa mass lesions, and is therefore recommended

Table 8. Brain Trauma Foundation: surgical management of traumatic brain injury (Bullock et al. [15]).

\section{Surgical management of depressed skull fractures}

Indications for surgery

- Patients with open (compound) skull fractures depressed greater than the thickness of the skull should undergo operative intervention to prevent infection

- Patients with open (compound) depressed skull fractures may be treated nonoperatively, if there is no clinical or radiographic evidence of dural penetration, significant intracranial hematoma, depression $>1 \mathrm{~cm}$, frontal sinus involvement, gross cosmetic deformity, wound infection, pneumencephalon or gross wound contamination

- Nonoperative management of closed (simple) depressed skull fractures is a treatment option

Timing

Early operation is recommended to reduce the incidence of infection

Methods

Elevation and debridement is recommended as the surgical method of choice.

Primary bone fragment replacement is a surgical option in the absence of wound infection at the time of surgery. All management strategies for open (compound) depressed fractures should include antibiotics

(1) the prophylactic DCE during evacuation of a mass lesion, based on clinical signs (neurologic state, CT scan, intraoperative swelling) instead of physiological parameters, and

(2) the therapeutic DCE

- when in patients not suffering from an evacuable mass lesion fatal pontine damage is already primarily present or
- when with protocol-driven management ICP becomes intractable.

Typically, therapeutic DCE is considered a last resort when staged application of medical interventions (including hypothermia and barbiturates) and ventricular CSF drainage have failed to control posttraumatic intracranial hypertension $[40,74,75,83,98,122]$ of $>30$ $\mathrm{mmHg}$ when associated with a CPP of $<70 \mathrm{mmHg}$, or 
when the ICP exceeded $35 \mathrm{mmHg}$ irrespective of CPP [122]. For Kontopoulos et al. [59] the indication for DCE included the appearance or deterioration on the CT scan of diffuse unilateral or bilateral brain swelling in correlation with clinical deterioration, dilatation of pupils, unresponsiveness to light, increase of ICP to $>30$ $\mathrm{mmHg}$ and/or reduction of CPP to $<60 \mathrm{mmHg}$ for a period $>15 \mathrm{~min}$ and failure to respond to maximal medical treatment mentioned above. In children, Ruf et al. [95] initiated DCE including dura opening in cases of a sustained increase in ICP $>20 \mathrm{mmHg}$ for $>30 \mathrm{~min}$ despite maximally intensified conservative therapy (optimized sedation and ventilation, barbiturates or mannitol). In rare cases therapeutic DCE is performed in order to decompress brain swelling due to diffuse axonal injury grade III according to Marshall et al. [73] diagnosed on initial CT scan. Coplin et al. [20] start DCE as the initial surgical intervention for STBI with horizontal midline shift greater than explained by a removable hematoma. Prophylactic DCE [81] is performed by refraining from reinserting the removed skull flap after evacuating a mass lesion (acute epidural hematoma, subdural hematoma or intraparenchymal lesion).

Polin et al. [89] argue that once the ICP reaches a sustained level $>40 \mathrm{mmHg}$, the chance for intervention before permanent neurologic devastation has passed. Similarly, if the patient is not operated on promptly, sustained damage from elevated ICP can preclude recovery.

\section{Technique}

There is considerable debate in terms of the nuances of the type and the extension of subtemporal decompression:

- fronto-parieto-temporal unilateral [81] versus bilateral;

- bifrontal with/without cutting through the superior sagittal sinus and the falx cerebri [57, 89, 118, 122];

- subtemporal decompression $[4,38]$;

- circumferential craniotomy [17].

Furthermore, the dura may be left intact, scarificated [90], sliced [79] or it may be augmented by a plasty [20]. In order to reduce venous congestion, the creation of vascular tunnels may be considered [22]. The DCE may also be combined with a temporal lobectomy $[66,85]$.

The most favorable effect seems to results from large fronto-subtemporo-parieto-occipital craniectomies combined with enlargement of the dura. It appears to be essential that the craniotomy extends down to the floor of the middle cranial fossa. Cranioplasty should be performed as earlier as edema has resolved [62].

\section{Complications}

Despite several series of this procedure reported in the literature, the complication rate of the operation is unclear. Patients may develop intracerebral or subdural hematomas [80] necessitating evacuation, injury to the cerebral cortex, reactive cerebral edema and brain herniation through the defect escalating the problems [19, $34,39]$. Shift of the brain with variable degrees of necrosis, CSF leaks, injury to the venous sinuses and infections have been reported. DCE seems to increase the incidence of hygromas and hydrocephalus $[40,89]$.

Rarely, a sinking scalp flap syndrome may develop, in which there is neurologic deterioration, thought to be related to the concavity of the skin flap and underlying brain tissue secondary to atmospheric pressure and also to the in-and-out displacement of the brain through the skull defect [97]. Care must be taken to avoid the sigmoid and superior sagittal sinuses to avoid complications related to thrombosis. Skull reconstruction itself is associated with infections and bone flap resorption after cranioplasty.

\section{Clinical Outcome}

The concern that DCE may save life at the expense of increasing the number of patients in vegetative state and severe disability appears not to be founded by the literature [86]. Based on the results of several different series [2, 26, 40, 59, 61, 81, 89], the outcome following DCE is very variable in the long term: mortality rate ranges from $11 \%$ to $59 \%$, a rate of "vegetative/severe disability" was observed in $11-41 \%$, and the rate of "recovery/moderate disability" was reported to be between $25 \%$ and $66 \%$.

Polin et al. [89] report that most of their failures consisted of heroic attempts to help patients recover from injuries from which they likely could not recover, operating on patients without severe ICP elevation whose poor neurologic states were likely secondary to diffuse axonal injury, or operating on patients with a GCS score of 3 .

The reason for these conflicting results are the heterogeneous character of the patient population, the different indications and starting points for treatment, as well as different surgical procedures. Larger studies employing standardized protocols for neurosurgical intervention are clearly warranted.

\section{Conclusion}

The effect of DCE on outcome has not been established. There is, however, evidence that the operation does favor- 
ably influence ICP, intracranial volume and midline shift and basal cisterns as a surrogate endpoint $[50,81,122]$.

The guidelines established by the American Association of Neurological Surgeons (AANS) [15] name $\mathrm{DCE}$ as the last of the second-tier therapeutic options. However, among second-tier measures, DCE leads to the fastest relief by immediate reduction of intracranial hypertension and has the lowest rate of complications (systemic side effects) as compared to other medical options, notably barbiturates and hypothermia $[18,93,94]$. Moreover, the procedure is simple and safe. In our opinion, DCE should therefore be the first among second-tier therapeutic options rather than the last one.

DCE is currently being applied in the management of TBI with a wide range of outcomes reported in the literature. The fact that there are several series in the literature which are supportive of DCE, with evidence that the operation does reduce ICP and that ICP is related to outcome, strongly supports proceeding with randomized studies.

Proof of a beneficial effect of DCE on outcome may only be obtained with prospective randomized trials. Such a study should not only consider the indication for DCE but also the time point and the technique with regard to the dimensions of the hemi- and bifrontal craniectomies. Currently, such a multicenter study is being planned (RESCUE-ICP, http://rescueicp.com).

\section{Overall Conlusion}

Because of the lack of class I und class II studies, there are no standards nor guidelines concerning

- the indication for CT scanning in patients suffering from MTBI,

- the surgical management of acute epidural hematomas, acute subdural hematomas, traumatic parenchymal lesions, posterior fossa mass lesions, as well as depressed skull fractures.

Therefore most recommendations are based on expert opinion and clinical experience, and satisfy criteria for so-called Class III recommendations or practice Options only.

Therefore, the general conclusions and recommendations created by the Brain Trauma Foundation [15] (evidence-based, where available) and the EBIC [72] (consensus- and expert opinion-based) are very similar.

Even if they do not meet class I or II standards, treatment protocols serve an important purpose, because they may improve management of TBI patients by promoting uniform decision-making.

\section{References}

1. Adelson PD, Bratton SL, Carney NA, et al. Guidelines for the acute medical management of severe traumatic brain injury in infants, children, and adolescents. Pediatr Crit Care Med 2003;4:1-71.

2. Albanese J, Leone $M$, Alliez JR, et al. Decompressive craniectomy for severe traumatic brain injury: evaluation of the effects at one year. Crit Care Med 2003;31:2535-8.

3. Alberico A, Ward J, Choi S, et al. Outcome after severe head injury. Relationship to mass lesions, diffuse injury, and ICP course in pediatric and adult patients. J Neurosurg 1987;67:648-56.

4. Alexander E, Ball MR, Laster DW. Subtemporal decompression: radiological observations and current surgical experience. Br J Neurosurg 1987:1:427-33.

5. American Academy of Pediatrics. The management of minor closed head injury in children. Committee on Quality Improvement, American Academy of Pediatrics. Commission on Clinical Policies and Research, American Academy of Family Physicians. Pediatrics 1999;104:1407-15.

6. American College of Surgeons Committee on Trauma. Advanced Trauma Life Support for doctors, 76 th edn. Chicago: American College of Surgeons, 2004:1-391.

7. Bagley RS, Harrington ML, Pluhar GE, et al. Effect of craniectomy/ durotomy alone and in combination with hyperventilation, diuretics, and corticosteroids on intracranial pressure in clinically normal dogs. Am J Vet Res 1996;57:116-9.

8. Bartlett J, Kett-White R, Mendelow AD, et al. Recommendations from the Society of British Neurological Surgeons. Br J Neurosurg 1998;12:349-52.

9. Becker DP, Miller JD, Ward JD, et al. The outcome from severe head injury with early diagnosis and intensive treatment. J Neurosurg 1977;47:491-502.

10. The Brain Trauma Foundation, the American Association of Neurological Surgeons: the Joint Section on Neurotrauma and Critical Care. Indications for intracranial pressure monitoring. J Neurotrauma 2000;17:479-91.

11. The Brain Trauma Foundation, the American Association of Neurological Surgeons: the Joint Section on Neurotrauma and Critical Care. Intracranial pressure treatment threshold. J Neurotrauma 2000;17:493-5.

12. Bricolo A, Pasut L. Exadural hematoma: toward zero mortality. A prospective study. Neurosurgery 1984;14:8-12.

13. Brown CV, Weng J, Oh D, et al. Does routine serial computed tomography of the head influence management of traumatic brain injury? A prospective evaluation. J Trauma 2004;57:939-43.

14. Bulger EM, Nathens AB, Rivara FP, et al. Management of severe head injury: institutional variations in care and effect on outcome. Crit Care Med 2002;30:1870-86.

15. Bullock MR, Chesnut RM, Ghajar J, et al. Surgical management of traumatic brain injury. New York: Brain Trauma Foundation, 2000.

16. Chesnut RM, Marshall LF. Management of head injury. Treatment of abnormal intracranial pressure. Neurosurg Clin N Am 1991; 2:267-84.

17. Clark K, Nash TM, Hutchison GC. The failure of circumferential craniotomy acute traumatic cerebral swelling. J Neurosurg 1968;29:367-71.

18. Clifton GL, Miller ER, Choi SC, et al. Lack of effect of induction of hypothermia after acute brain injury. N Engl J Med 2001;344: 556-63.

19. Cooper PR, Hagler H, Clark WK, et al. Enhancement of experimental cerebral edema after decompressive craniectomy: implications for the management of severe head injuries. Neurosurgery 1979;4:296-300.

20. Coplin WM, Cullen NK, Policherla PN, et al. Safety and feasibility of craniectomy with duraplasty as the initial surgical interven- 
tion for severe traumatic brain injury. J Trauma 2001;50:1050-9.

21. Cranshaw J, Hughes G, Clancy M. Computerised tomography and acute traumatic head injury: time for change? J Accid Emerg Med 1996;13:80-5.

22. Csokay A, Egyud L, Nagy L, et al. Vascular tunnel creation to improve the efficacy of decompressive craniotomy in post-traumatic cerebral edema and ischemic stroke. Surg Neurol 2002;57:126-9.

23. Cushing $\mathrm{H}$. The establishment of cerebral hernia as a decompressive measure for inaccessible brain tumor: with the description of intramuscular methods of making the bone defect in temporal and occipital regions. Surg Gynecol Obstet 1905;1:297-314.

24. Czosnyka M, Pickard JD. Monitoring and interpretation of intracranial pressure. J Neurol Neurosurg Psychiatry 2004;75:813-21.

25. Dam Hieu P, Sizun J, Person H, et al. The place of decompressive surgery in the treatment of uncontrollable post-traumatic intracranial hypertension in children. Childs Nerv Syst 1996;12:270-5.

26. De Luca GP, Volpin L, Fornezza U, et al. The role of decompressive craniectomy in the treatment of uncontrollable post-traumatic intracranial hypertension. Acta Neurochir Suppl (Wien) 2000;76:401-4.

27. Dent DL, Croce MA, Menke PG, et al. Prognostic factors after acute subdural hematoma. J Trauma 1995;39:36-43.

28. Dunham CM, Coates S, Cooper C. Compelling evidence for discretionary brain computed tomographic imaging in those patients with mild cognitive impairment after blunt trauma. J Trauma 1996;41:679-86.

29. Eisenberg HM, Frankowski RF, Contant CF, et al. High-dose barbiturate control of elevated intracranial pressure in patients with severe head injury. J Neurosurg 1988;69:15-23.

30. Eisenberg HM, Gary HE Jr, Aldrich EF, et al. Initial CT findings in 753 patients with severe head injury. A report from the NIH Traumatic Coma Data Bank. J Neurosurg 1990;73:688-98.

31. Fabbri A, Servadei F, Marchesini G, et al. Prospective validation of a proposal for diagnosis and management of patients attending the emergency department for mild head injury. J Neurol Neurosurg Psychiatry 2004;75:410-6.

32. Fakhry SM, Trask AL, Waller MA, et al., Neurotrauma Task Force. Management of brain-injured patients by an evidence-based medicine protocol improves outcomes and decreases hospital charges. J Trauma 2004;56:492-9, discussion 499-500.

33. Figaji AA, Fieggen AG, Peter JC. Early decompressive craniotomy in children with severe traumatic brain injury. Childs Nerv Syst 2003;19:666-73.

34. Gaab M, Knoblich OE, Fuhrmeister U, et al. Comparison of the effects of surgical decompression and resection of local edema in the therapy of experimental brain trauma. Childs Brain 1979;5: 484-98.

35. Gaab MR, Rittierodt M, Lorenz M, et al. Traumatic brain swelling and operative decompression: a prospective investigation. Acta Neurochir Suppl (Wien) 1990;51:326-8.

36. Ghajar JB. A guide for ventricular catheter placement. Technical note. J Neurosurg 1985;63:985-6.

37. Giannoudis PV, Veysi VT, Pape HC, et al. When should we operate on major fractures in patients with severe head injuries? Am J Surg 2002;183:261-7.

38. Gower DJ, Lee KS, McWhorter JM. Role of subtemporal decompression in severe closed head injury. Neurosurgery 1988;23: 417-22.

39. Grande PO, Asgeirsson B, Nordstrom CH. Volume-targeted therapy of increased intracranial pressure: the Lund concept unifies surgical and non-surgical treatments. Acta Anaesthesiol Scand 2002;46:929-41.

40. Guerra WK, Gaab MR, Dietz H, et al. Surgical decompression for traumatic brain swelling: indications and results. J Neurosurg 1999;90:187-96.
41. Harrington ML, Bagley RS, Moore MP, et al. Effect of craniectomy, durotomy, and wound closure on intracranial pressure in healthy cats. AJNR Am J Neuroradiol 1996;57:1659-61.

42. Hase U, Reulen HJ, Meinig G, et al. The influence of the decompressive operation on the intracranial pressure and the pressure-volume relation in patients with severe head injuries. Acta Neurochir (Wien) 1978;45:1-13.

43. Haselsberger K, Pucher R, Auer LM. Prognosis after acute subdural or epidural haemorrhage. Acta Neurochir (Wien) 1988;90:111-6.

44. Hatashita S, Hoff JT. The effect of craniectomy on the biomechanics of normal brain. J Neurosurg 1987;67:573-8.

45. Hejazi N, Witzmann A, Fae P. Unilateral decompressive craniectomy for children with severe brain injury. Report of seven cases and review of the relevant literature. Eur J Pediatr 2002;161:99-104.

46. Hickman KM, Mayer BL, Muwaswes M. Intracranial pressure monitoring: review of risk factors associated with infection. Heart Lung 1990;19:84-90.

47. Hill DA, Abraham KJ, West RH. Factors affecting outcome in the resuscitation of severely injured patients. Aust N Z J Surg 1993;63:604-9.

48. Hofman PA, Nelemans P, Kemerink GJ, et al. Value of radiological diagnosis of skull fracture in the management of mild head injury: metaanalysis. J Neurol Neurosurg Psychiatry 2000;68:416-22.

49. Holloway KL, Barnes T, Choi S, et al. Ventriculostomy infections: the effects of monitoring duration and catheter exchange in 584 patients. J Neurosurg 1996;85:419-26.

50. Hutchinson PJ, Kirkpatrick PJ. Decompressive craniectomy in head injury. Curr Opin Crit Care 2004;10:101-4.

51. Ingebrigtsen T, Romner B, Kock-Jensen C. Scandinavian guidelines for initial management of minimal, mild, and moderate head injuries. The Scandinavian Neurotrauma Committee. J Trauma 2000;48:760-6.

52. Jaeger $M$, Soehle $M$, Meixensberger J. Effects of decompressive craniectomy on brain tissue oxygen in patients with intracranial hypertension. J Neurol Neurosurg Psychiatry 1993;74:513-5.

53. Jeret JS, Mandell M, Anziska B, et al. Clinical predictors of abnormality disclosed by computed tomography after mild head trauma. Neurosurgery 1993;32:9-15.

54. Jourdan C, Convert J, Mottolese C, et al. Evaluation of the clinical benefit of decompression hemicraniectomy in intracranial hypertension not controlled by medical treatment. Neurochirurgie 1993;39:304-10.

55. Juul N, Morris GF, Marshall SB, et al. Intracranial hypertension hypertension and cerebral perfusion pressure: influence on neurological deterioration and outcome in severe head injury - the Executive Committee of the International Selfotel Trial L. J Neurosurg 2000;92:1-6.

56. Kaups KL, Davis JW, Parks SN. Routinely repeated computed tomography after blunt head trauma: does it benefit patients? J Trauma 2004;56:475-80.

57. Kjellberg RN, Prieto AJ. Bifrontal decompressive craniotomy for massive cerebral edema. J Neurosurg 1971;34:488-93.

58. Klauber MR, Marshall LF, Luerssen TG, et al. Determinants of head injury mortality: importance of the low risk patient. Neurosurgery 1989;24:31-6.

59. Kontopoulos V, Foroglou N, Patsalas J, et al. Decompressive craniectomy for the management of patients with refractory hypertension: should it be reconsidered? Acta Neurochir (Wien) 2002;144:791-6.

6o. Kotwica Z, Brzezinski J. Acute subdural haematoma in adults: an analysis of outcome in comatose patients. Acta Neurochir (Wien) 1993;121:95-9.

61. Kunze E, Meixensberger J, Janka $M$, et al. Decompressive craniectomy in patients with uncontrollable intracranial hypertension. Acta Neurochir Suppl (Wien) 1998;71:16-8. 
62. Kuo JR, Wang CC, Chio CC, et al. Neurological improvement after cranioplasty - analysis by transcranial Doppler ultrasonography. J Clin Neurosci 2004;11:486-9.

63. Lane PL, Skoretz TG, Doig G, et al. Intracranial pressure monitoring and outcomes after traumatic brain injury. Can J Surg 2000; 43:442-8.

64. Lang EW, Chesnut RM. Intracranial pressure. Monitoring and management. Neurosurg Clin N Am 1994;5:573-605.

65. Langfitt TW, Tannanbaum HM, Kassel NF. The etiology of acute brain swelling following experimental head injury. J Neurosurg 1966;24:47-56.

66. Lee EJ, Chio CC, Chen HH. Aggressive temporal lobectomy for uncal herniation in traumatic subdural hematoma. J Formos Med Assoc 1995;94:341-5.

67. Lee LA, Sharar SR, Lam AM. Perioperative head injury management in the multiply injured trauma patient. Int Anesthesiol Clin 2002;40:31-52.

68. Livingston DH, Lavery RF, Passannante MR, et al. Emergency department discharge of patients with a negative cranial computed tomography scan after minimal head injury. Ann Surg 2000; 232:126-32.

69. Livingston DH, Loder PA, Koziol J, et al. The use of CT scanning to triage patients requiring admission following minimal head injury. J Trauma 1991;31:483-7.

70. Lobato RD, Gomez PA, Alday R, et al. Sequential computerized tomography changes and related final outcome in severe head injury patients. Acta Neurochir (Wien) 1997;139:385-91.

71. Lobato RD, Rivas JJ, Gomez PA, et al. Head-injured patients who talk and deteriorate into coma. Analysis of 211 cases studied with computerized tomography. J Neurosurg 1991;75:256-61.

72. Maas AI, Dearden M, Teasdale GM, et al. EBIC-guidelines for management of severe head injury in adults. European Brain Injury Consortium. Acta Neurochir (Wien) 1997;139:286-94.

73. Marshall LF, Marshall SB, Klauber MR, et al. A new classification of head injury based on computerized tomography. J Neurosurg 1991;75:Suppl 1:14-20.

74. Meier U, Grawe A. The importance of decompressive craniectomy for the management of severe head injuries. Acta Neurochir Suppl (Wien) 2003;86:367-71.

75. Meier U, Zeilinger FS, Henzka O. The use of decompressive craniectomy for the management of severe head injuries. Acta Neurochir Suppl (Wien) 2000;76:475-8.

76. Mendelow AD, Teasdale G, Jennett $B$, et al. Risks of intracranial haematoma in head injured adults. Br Med J [Clin Res] 1983; 287:1173-6.

77. Miller JD, Becker DP, Ward JD, et al. Significance of intracranial hypertension in severe head injury. J Neurosurg 1977;47:503-16.

78. Miller JD, Butterworth JF, Gudeman SK, et al. Further experience in the management of severe head injury. J Neurosurg 1981; 54:289-99.

79. Mitchell P, Tseng M, Mendelow AD. Decompressive craniectomy with lattice duraplasty. Acta Neurochir (Wien) 2004;146:159-6o.

8o. Moody RA, Ruamsuke S, Mullan SF. An evaluation of decompression in experimental head injury. J Neurosurg 1968;29:586-90.

81. Muench E, Horn P, Schürer L, et al. Management of severe traumatic brain injury by decompressive craniectomy. Neurosurgery 2000;47:315-23.

82. Muizelaar JP, Marmarou A, Ward JD, et al. Adverse effects of prolonged hyperventilation in patients with severe head injury: a randomized clinical trial. J Neurosurg 1991;75:731-9.

83. Mussack T, Wiedemann E, Hummel T, et al. Secondary decompression trepanation in progressive post-traumatic brain edema after primary decompressive craniotomy. Unfallchirurg 2003;106: $815-25$.
84. Narayan RK, Kishoure PRS, Becker DP, et al. Intracranial pressure: to monitor or not to monitor. A review of our experiences with severe head injury. J Neurosurg 1982;56:650-9.

85. Nussbaum ES, Wolf AL, Sebring L, et al. Complete temporal lobectomy for surgical resuscitation of patients with transtentorial herniation secondary to unilateral hemispheric swelling. Neurosurgery 1991;29:62-6.

86. Patel HC, Menon DK, Tebbs S, et al. Specialist neurocritical care and outcome from head injury. Intensive Care Med 2002;28:547-53.

87. Piek J. Decompressive surgery in the treatment of traumatic brain injury. Curr Opin Crit Care 2002;8:134-8.

88. Piek J, Chesnut RM, Marshall LF, et al. Extracranial complications of severe head injury. J Neurosurg 1992;77:901-7.

89. Polin RS, Shaffrey ME, Bogaev CA, et al. Decompressive bifrontal craniectomy in the treatment of severe refractory posttraumatic cerebral edema. Neurosurgery 1997;41:84-92.

90. Price DJ, Czosnyka Z, Kotas G, et al. Improving compensatory reserve by craniectomy and dural scarification. In: Nagai H, Kamiya K, Ishii S, eds. Intracranial pressure IX. New York: Springer, 1994:464-5.

91. Ransohoff J, Benjamin MV, Gage EL, et al. Hemicraniectomy in the management of acute subdural hematoma. J Neurosurg 1971; 34:70-6.

92. Reithmeier T, Speder B, Pakos P, et al. Delayed bilateral craniectomy for treatment of traumatic brain swelling in children: case report and review of the literature. Childs Nerv Syst 2005;21:249-53.

93. Roberts I. Barbiturates for acute traumatic brain injury. Cochrane Database Syst Rev 2000;2:CDoo0033.

94. Robertson CS, Valadka AB, Hannay HJ, et al. Prevention of secondary ischaemic insults after severe head injury. Crit Care Med 2004;27:2086-95.

95. Ruf B, Heckmann M, Schroth I, et al. Early decompressive craniectomy and duraplasty for refractory intracranial hypertension in children: results of a pilot study. Crit Care 2003;7:R133-8.

96. Sakas DE, Bullock MR, Teasdale GM, et al. One-year outcome following craniotomy for traumatic hematoma in patients with fixed dilated pupils. J Neurosurg 1995;82:961-5.

97. Schiffer J, Gur R, Nisim U, et al. Symptomatic patients after craniectomy. Surg Neurol 1997;47:231-7.

98. Schneider GH, Bardt T, Lanksch WR, et al. Decompressive craniectomy following traumatic brain injury: ICP, CPP and neurological outcome. Acta Neurochir Suppl (Wien) 2002;81:77-9.

99. Seelig JM, Becker DP, Miller JD, et al. Traumatic acute subdural hematoma: major mortality reduction in comatose patients treated within four hours. N Engl J Med 1981;304:1511-58.

100. Servadei F, Teasdale G, Merry G, Neurotraumatology Committee of the World Federation of Neurosurgical Societies. Defining acute mild head injury in adults: a proposal based on prognostic factors, diagnosis, and management. J Neurotrauma 2001; 18:657-64.

101. Signorini DF, Andrews PJ, Jones PA, et al. Adding insult to injury: the prognostic value of early secondary insults for survival after traumatic brain injury. J Neurol Neurosurg Psychiatry 1999; 66:26-31.

102. Simon B, Letourneau P, Vitorino E, et al. Pediatric minor head trauma: indications for computed tomographic scanning revisited. J Trauma 2001;51:231-7.

103. The Society of British Neurological Surgeons. Guidelines for the initial management of head injuries: recommendations from the Society of British Neurological Surgeons. Br J Neurosurg 1998; 12:349-52.

104. Stein SC. Management of minor closed head injury. Neurosurg 0 1996;6:108-15.

105. Stiell IG, Wells GA, Vandemheen K, et al. The Canadian CT Head Rule for patients with minor head injury. Lancet 2001;357:1391-6. 
106. Stocchetti N, Rossi S, Buzzi F, et al. Intracranial hypertension in head injury: management and results. Int Care Med 1999;25:371-6.

107. Tabori U, Kornecki A, Sofer S, et al. Repeat computed tomographic scan within 24-48 hours of admission in children with moderate and severe head trauma. Crit Care Med 2000;28:840-4.

108. Taylor A, Butt W, Rosenfeld J, et al. A randomized trial of very early decompressive craniectomy in children with traumatic brain injury and sustained intracranial hypertension. Childs Nerv Syst 2001;17:154-62.

109. Teasdale G, Murray G, Anderson E, et al. Risks of acute traumatic intracranial haematoma in children and adults: implications for managing head injuries. BMJ 1990;300:363-7.

110. Teasdale GM, Jennett B. Assessment of coma and impaired consciousness. A practical scale. Lancet 1974;2:81-4.

111. Thillainayagam K, MacMillan R, Mendelow AD, et al. How accurately are fractures of the skull diagnosed in an accident and emergency department? Injury 1987;18:319-21.

112. Thiruppathy SP, Muthukumar N. Mild head injury: revisited. Acta Neurochir (Wien) 2004;146:1075-82.

113. Thurman DGJ. Trends in hospitalization associated with traumatic brain injury. JAMA 1999;282:954-7.

114. Toutant SM, Klauber MR, Marshall LF. Absent or compressed basal cisterns on first CT scan: ominous predictors of outcome in severe head injury. J Neurosurg 1984;61:691-4.

115. Twijnstra A, Brouwer OF, Keyser A, et al. Richtlijnen voor de diagnostiek en behandeling van patienten met licht schedel-hersenletsel. Maastricht: Commissie Kwaliteitsbevordering van de Nederlandse Vereniging voor Neurologie 2001:1-26.

116. Ucar T, Akyuz M. Management of severe traumatic brain injury by decompressive craniectomy. Neurosurgery 2001;49:1022.

117. Van Dongen KJ, Braakman R, Gelpke GJ. The prognostic value of computerized tomography in comatose head-injured patients. J Neurosurg 1983:59:951-7.

118. Venes JL, Collins WF. Bifrontal decompressive craniectomy in the management of head trauma. J Neurosurg 1975;42:429-33.

119. Viola L, Zotta D, Martino V, et al. Minor head injuries: one year experience according to the new Italian guideline. Acta Neurochir (Wien) 2000;142:1281-5.
120. Vilke GM, Chan TC, Guss DA. Use of a complete neurological examination to screen patients for significant intracranial abnormalities in minor head injury. Am J Emerg Med 2000;18:159-63.

121. Vos PE, Battistin L, Birbamer G, et al. EFNS guideline on mild traumatic brain injury: report of an EFNS task force. Eur J Med 2000; 9:207-19.

122. Whitfield PC, Patel $\mathrm{H}$, Hutchinson PJ, et al. Bifrontal decompressive craniectomy in the management of posttraumatic intracranial hypertension. Br J Neurosurg 2001;15:500-7.

123. Wilberger JE, Harris M, Diamond DL. Acute subdural hematoma: morbidity, mortality, and operative timing. J Neurosurg 1991; 74:212-8.

124. Winfield JA, Rosenthal P, Kanter RK, et al. Duration of intracranial pressure monitoring does not predict daily risk of infective complications. Neurosurgery 1993;33:424-31.

125. Woolf SH. Practice guidelines, a new reality in medicine. II. Methods of developing guidelines. Arch Intern Med 1992;152:946-52.

126. Yoo DS, Kim DS, Cho KS, et al. Ventricular pressure monitoring during bilateral decompression with dural expansion. J Neurosurg 1999;91:953-9.

127. Zhong J, Dujovny M, Park HK, et al. Advances in ICP monitoring techniques. Neurol Res 2003;25:339-50.

\section{Address for Correspondence}

Professor Hans-Georg Imhof, MD

Department of Neurosurgery

University Hospital of Zurich

Rämistraße 100

8091 Zürich

Switzerland

Phone (+41/44) 255-1111, Fax -5741

e-mail: hans-georg.imhof@usz.ch 